University of Nebraska - Lincoln

DigitalCommons@University of Nebraska - Lincoln

$1-14-2001$

\title{
Quasistationary Stabilization of the Decay of a Weakly-Bound Level and Its Breakdown in a Strong Laser Field
}

N. L. Manakov

Voronezh State University, manakov@phys.vsu.ru

M. V. Frolov

Voronezh State University, Russia

Bogdan Borca

Institute for Space Sciences, Bucharest, Romania

Anthony F. Starace

University of Nebraska-Lincoln, astarace1@unl.edu

Follow this and additional works at: https://digitalcommons.unl.edu/physicsstarace

Part of the Physics Commons

Manakov, N. L.; Frolov, M. V.; Borca, Bogdan; and Starace, Anthony F., "Quasistationary Stabilization of the Decay of a Weakly-Bound Level and Its Breakdown in a Strong Laser Field" (2001). Anthony F. Starace Publications. 116.

https://digitalcommons.unl.edu/physicsstarace/116

This Article is brought to you for free and open access by the Research Papers in Physics and Astronomy at DigitalCommons@University of Nebraska - Lincoln. It has been accepted for inclusion in Anthony F. Starace Publications by an authorized administrator of DigitalCommons@University of Nebraska - Lincoln. 
Published in Super-Intense Laser-Atom Physics, edited by Bernard Piraux and Kazimierz Rzążewski. NATO Science Series:

Series II: Mathematics, Physics and Chemistry - Vol. 12. Dordrecht / Boston / London: Kluwer Academic Publishers, 2001.

Proceedings to the NATO Advanced Research Workshop on Super-Intense Laser-Atom Physics, Han-sur-Lesse, Belgium, September 24-30, 2000.

Copyright (c) 2001 Kluwer Academic Publishers. Used by permission.

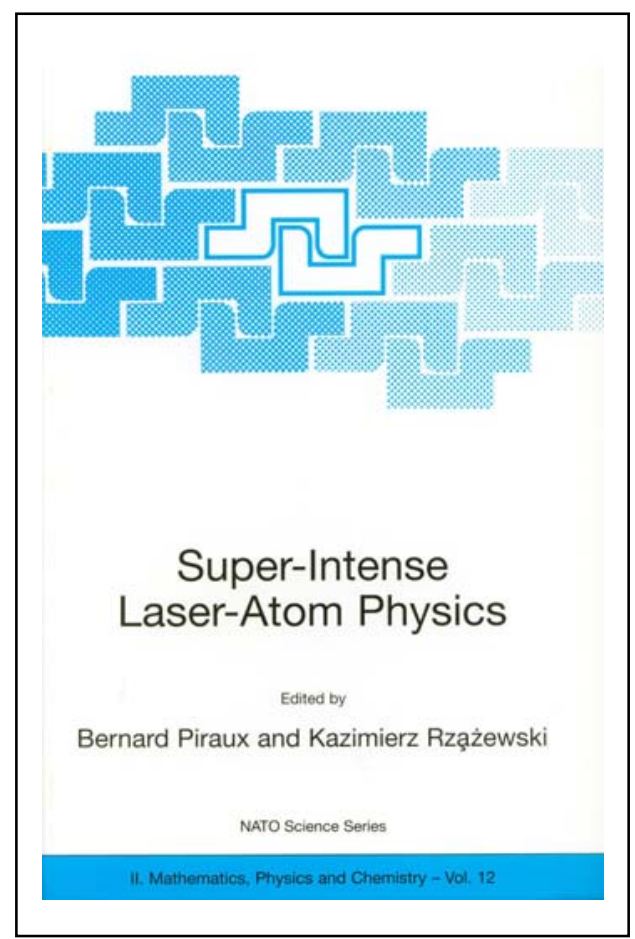




\title{
QUASISTATIONARY STABILIZATION OF THE DECAY OF A WEAKLY-BOUND LEVEL AND ITS BREAKDOWN IN A STRONG LASER FIELD
}

\author{
N. L. MANAKOV, M. V. FROLOV \\ Voronezh State University, 394693 Voronezh, Russia \\ AND \\ BOGDAN BORCA, ${ }^{*}$ ANTHONY F. STARACE \\ Department of Physics and Astronomy, The University of Nebraska \\ 116 Brace Laboratory, Lincoln, NE 68588-0111, USA
}

\section{Introduction}

Although it was pointed out about 10 years ago [1,2] that an atomic decay rate might decrease as the intensity of a high-frequency laser field increases, there still does not exist any complete understanding of either the physical origin of this interesting nonlinear phenomenon or its dependence on the atomic and field parameters. Essentially, the problem consists in that the phenomenon requires a major modification of the standard picture of photoeffect in a strong laser field. In Ref. [1] the origin of this stabilization is related to a particular distortion of an atomic potential by an intense monochromatic high-frequency field. This phenomenon is called adiabatic or quasistationary stabilization (QS). For the case of Rydberg levels, another (interference) mechanism of QS was suggested [2]. Both theories predict an unlimited decrease of the decay rate (or of the width $\Gamma$ of an atomic level, i.e., of the imaginary part of the complex quasienergy, $\epsilon=\operatorname{Re} \epsilon-i \Gamma / 2)$ ) as the laser field amplitude increases. In recent years the idea of "dynamic stabilization" (DS) [3] has become popular. It originates from the pulse form of a laser field rather than from any intrinsic property of the atom in a strong monochromatic field. Within this model the numerous simulations point also to the possibility of a breakdown of stabilization for the case of superintense short laser pulses [4]. However, a recent paper [5], using the quasistationary quasienergy states (QQES) as an adiabatic basis for the laser pulse has shown that DS has the same (quasistationary) origin 
as QS. Finally, a number of authors deny the existence of stabilization, in particular, of QS for ionization from a short-range potential [6] (see also [7]) and of DS in pulsed fields [8]. Obviously, these controversies and ambiguities are caused by the complexity of the numerical solution of the Cauchy problem for the time-dependent Schrödinger equation in a strong field and by the absence of analyses for exactly solvable analytical models. We analyze below (see also [9]) the exactly solvable problem of an electron in a three-dimensional, short-range potential and consider the two questions: does a QS-like behavior of the decay rate exist for this model, and, if so, is there an upper intensity limit of the QS regime?

\section{Basic equations and numerical results}

We consider the quasistationary decay of a bound state in a monochromatic laser field with electric vector

$$
\mathbf{F}(t)=\frac{F}{\sqrt{1+\eta^{2}}}\{\cos \omega t, \eta \sin \omega t, 0\}, \quad-1 \leq \eta \leq+1,
$$

and intensity $I=c F^{2} / 8 \pi$ based on the QQES approach [10] for the exactly solvable model of a 3 -dimensional, short-range $(\delta-)$ potential having one bound state with energy $E_{0}$. In this model, the complex quasienergy $\epsilon$ is the eigenvalue of a one-dimensional integral equation for the periodic (in time) function $\varphi_{\epsilon}(\tau=\omega t)$, which determines the asymptotic behavior of $\Phi_{\epsilon}(\mathbf{r}, t)$ at origin $(r \rightarrow 0)[11]:$

$$
\begin{aligned}
& \left(\sqrt{\mathcal{E}_{F}-\epsilon}-1\right) \varphi_{\epsilon}(\tau)=\sqrt{\frac{\omega}{4 \pi i}} \int_{0}^{\infty} \frac{d \tau^{\prime}}{\tau^{\prime 3 / 2}} e^{-i\left(\mathcal{E}_{F}-\epsilon\right) \tau^{\prime} / \omega} \times \\
& \quad \times\left\{\varphi_{\epsilon}\left(\tau-\tau^{\prime}\right) e^{4 i \Delta \frac{\sin ^{2} \tau^{\prime} / 2}{\tau^{\prime}}\left[1-l \cos \left(2 \tau-\tau^{\prime}\right)\right]}-\varphi_{\epsilon}(\tau)\right\} .
\end{aligned}
$$

This equation involves the linear polarization degree of the laser field, $l=$ $\left(1-\eta^{2}\right) /\left(1+\eta^{2}\right)$, as well as the characteristic dimensionless parameters of the problem: $\hbar \omega /\left|E_{0}\right|$ and the ratio of the ponderomotive energy of a free electron, $(e F)^{2} /\left(4 m \omega^{2}\right)$, to either the photon energy, $\Delta=(e F)^{2} /\left(4 m \hbar \omega^{3}\right)$, or to $\left|E_{0}\right|, \mathcal{E}_{F}=(e F)^{2} /\left(4 m \omega^{2}\left|E_{0}\right|\right)=\Delta\left(\hbar \omega /\left|E_{0}\right|\right)$. In scaled units, $\mathcal{E}_{F}=$ $F^{2} / \omega^{2}$ and $\Delta=F^{2} / \omega^{3}$. (The Keldysh parameter, $\gamma=\sqrt{2 m\left|E_{0}\right|} \omega / e F$, in our units is: $\gamma=\omega / \sqrt{2} F$.)

For the case of circular polarization of $\mathbf{F}(t)$ (i.e., $\eta= \pm 1$ ), $\varphi_{\epsilon}(\tau) \sim$ const, in which case Eq. (1) reduces to the transcendental equation [12]:

$$
\sqrt{\mathcal{E}_{F}-\epsilon}-1=\sqrt{\frac{\omega}{4 \pi i}} \int_{0}^{\infty} \frac{d \tau}{\tau^{3 / 2}} e^{-i\left(\mathcal{E}_{F}-\epsilon\right) \tau / \omega}\left\{e^{4 i \Delta \frac{\sin ^{2} \tau / 2}{\tau}}-1\right\} .
$$


Since $\operatorname{Im} \epsilon<0$, the integrals in Eqs. (1) and (2) are formally divergent at their upper limit and hence must be understood as an analytical continuation of complex $\epsilon$ from the upper half-plane. For this analytical continuation we use the following relation,

$$
\int_{0}^{\infty} \frac{\mathrm{d} \tau}{\tau^{1 / 2}} \mathrm{e}^{-\mathrm{i} \alpha \tau} f(\tau)=\frac{1}{\sqrt{4 \pi \mathrm{i}}} \int_{-\infty}^{\infty} \frac{\mathrm{d} k}{\sqrt{\alpha+k}} \int_{-\infty}^{\infty} \mathrm{d} x \mathrm{e}^{\mathrm{i} k x} f(x),
$$

where the double integral is convergent for any $\alpha=\left(\mathcal{E}_{F}-\epsilon\right) / \omega$. Eq. (3) is a key result for numerical calculations involving strong fields. Note that a number of authors dealing with the general QQES formalism for the $\delta$ - model potential [11] (see, e.g., [13]) remove the divergence of integrals of the kind (3) by the (approximate) substitution $\epsilon \approx E_{0}=-1$, which is obviously incorrect for strong fields. In particular, it was what led to the incorrect results in Fig. 5 of Ref. [6] and, as a result, to the incorrect statement in Ref. [6] regarding the absence of the QS regime for short-range potentials. Although a number of other authors have performed acurate numerical calculations of $\Gamma$ for the $\delta$-potential (for predominantly circular (e.g., [14]), but also linear [15] polarization), they do not analyze the results in the context of stabilization.

In Fig. 1 we present our numerical results for $\Gamma(F)$ at a scaled frequency $\omega=1.55$, which corresponds to the Nd:YAG laser frequency for the case of the $\mathrm{H}^{-}$-ion (with $\left|E_{0}\right| \approx 0.752 \mathrm{eV}$ ). One sees that for not too high (but nonperturbative) $F$ up to $F=F_{c r}, \Gamma(F)$ demonstrates the typical QS-behavior (cf. Fig. 4 for the H-atom in [16]). The decrease of $\Gamma(F)$ in the QS domain is more pronounced for the case of linear polarization. In contrast with QS-calculations for the Coulomb potential, Fig. 1 shows a sharp breakdown of QS (i.e., a sharp rise of $\Gamma(F)$ ) beginning at a critical field, $F=F_{c r}$, which does not depend on the polarization (see Eq. (4) below). Fig. 2 gives the $\omega$-dependence of $\Gamma(F)$ for frequencies $\omega>1$ for the case of circular polarization. Both the size of the interval of QS (in $F$ ) and the magnitude of the decay rate $\Gamma$ in the QS regime increase with increasing $\omega$. Moreover, we find that at fixed $F$, for $F \lesssim F_{c r}, \Gamma(\omega)$ is approximated by the power law, $\Gamma(\omega) \sim \omega^{\alpha}$ with $\alpha \approx 3.92$. This result is in reasonable agreement with the value $\alpha=4$ given by the high-frequency theory of QS for a circularly-polarized field [1].

Concerning the breakdown of QS we note firstly that near the point $F=F_{c r}$ there is a narrow interval of fields, $F \leq F_{c r}$, in which the width $\Gamma$ exhibits an irregular behavior (see Fig. 2). An exact numerical calculation of $\Gamma$ in this interval is difficult. For a more thorough analysis of $\Gamma$ at $F \sim$ $F_{c r}$ we performed nonperturbative calculations of partial widths, $\Gamma^{(n)}(F)$, corresponding to the absorption of a fixed number, $n$, of photons. Since 


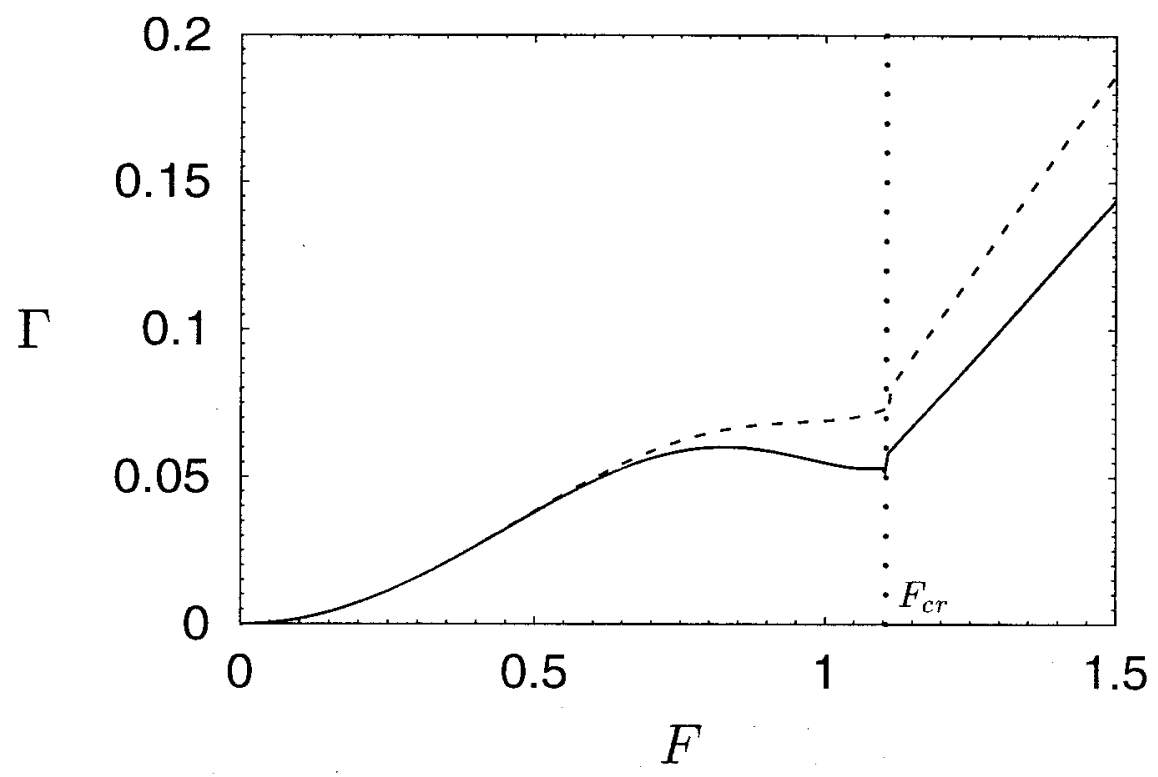

Figure 1. Dependence of $\Gamma$ on $F$ for linear (solid curve) and circular (dashed curve) polarizations of a laser field with $\omega=1.55$. The dotted vertical line denotes $F_{c r}$.

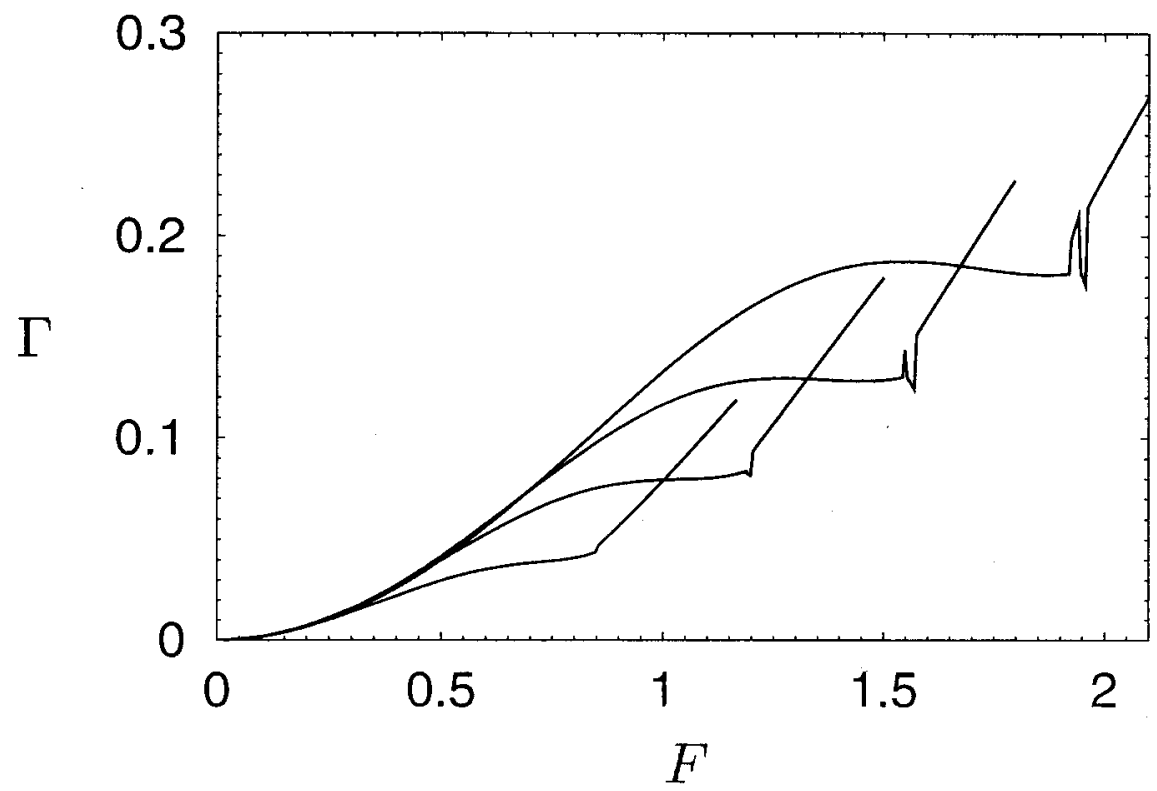

Figure 2. Dependence of $\Gamma$ on $F$ for a circularly polarized laser field having $\omega>1$. The curves (bottom to top) correspond to $\omega=1.4,1.6,1.8$, and 2.0 . 
the available space does not permit a detailed discussion, we note only that in the vicinity of $F_{c r}$ the one-photon width, $\Gamma^{(1)}$ (which corresponds to the photoelectron energy $E_{p}=\operatorname{Re} \epsilon-\mathcal{E}_{F}+\omega$ and almost completely determines the total width $\Gamma$ up to the midpoint of the QS interval), has a deep minimum. Thus the major contribution to $\Gamma$ in the breakdown domain stems from (interfering) channels of the direct $n$-photon above-threshold decay. Assuming that the breakdown of QS originates from the closeness of the direct photoionization channel, the estimation of $F_{c r}$ may be obtained from the relation $\operatorname{Re} \epsilon-\mathcal{E}_{F}+\omega=0$. Neglecting the Stark-shift (which is small for $\omega \sim 1$ ) one obtains

$$
F_{c r .} \approx \omega \sqrt{\omega-1}
$$

This estimation is in excellent agreement with values of $F_{c r}$ generated from the numerical calculations. A slight lowering of the calculated $F_{c r}$ with increasing $\omega$ compared to that in Eq. (4) is caused by the Stark-shift.

\section{Analytical results for circular polarization}

These results on the breakdown of stabilization and on the magnitude of the critical field $F_{c r}$ have important physical consequences, implying, in particular, the non-existence of the so-called Death Valley [1] (i.e., of a global deep minimum in the $F$-dependence of the lifetime of a quasistationary level) for a weakly-bound system in a strong monochromatic field. Hence to justify our numerical results and their physical interpretation, we present below some analytical considerations. It is well-known that for the case of circular polarization, $\epsilon$ may be obtained as the (complex) eigenvalue of the stationary QQES-Hamiltonian (see, e.g., [10]) in a coordinate frame rotating with the frequency $\omega$,

$$
\mathcal{H}_{\text {rot. }}(\mathbf{r})=-\nabla_{\mathbf{r}}^{2}+U(\mathbf{r})+F x \pm \omega \hat{L}_{z}
$$

where $\hat{\mathbf{L}}$ is the orbital angular momentum operator. We take into account the operator $\omega \hat{L}_{z}$ using perturbation theory (PT) in a basis of quasistationary states of an electron in the $\delta$-potential, $U(\mathbf{r})=4 \pi \delta(\mathbf{r})(\partial / \partial r) r$, and in a static field F. ( For PT for quasistationary states see, e.g., [17], and for a convenient form for the Green's function of our problem in terms of regular and irregular Airy functions, $\mathrm{Ai}(x)$ and $\mathrm{Bi}(x)$, as well as for techniques for PT calculations to second order, see [18].) The result for $\epsilon$ (taking into account corrections of order $\omega^{2}$ ) is:

$$
\epsilon=E-\frac{\omega^{2}}{360 F^{2 / 3}} \frac{I^{(4)}(\xi)}{I(\xi)}, \quad \xi=-\frac{E}{F^{2 / 3}}
$$


where $E$ is the exact (complex) energy of the quasistationary state in a static field F. $E$ is determined as the root of the transcendental equation:

$$
1+\pi F^{1 / 3} J\left(-E F^{-2 / 3}\right)=0,
$$

where $J(x)=\operatorname{Ai}^{\prime}(x) \mathrm{Ci}^{\prime}(x)-x \mathrm{Ai}(x) \mathrm{Ci}(x) ; \mathrm{Ci}(x)=\mathrm{Bi}(x)+\mathrm{iAi}(\dot{\mathrm{x}}) ; I(x)=$ $\mathrm{Ai}(x) \mathrm{Ci}(x) ; I^{(4)}(x)=\mathrm{d}^{4} I(x) / \mathrm{d} x^{4}$. (For an analysis of the function $E=$ $E(F)$, see [18].) Eqs. (5) and (6) do not assume that $F$ is small. Thus the known properties of the Airy functions allow a simple analysis of the applicability of $\mathrm{PT}$ in the operator $\omega \hat{L}_{z}$ (i.e., for the conditions under which the correction of order $\omega^{2}$ in Eq. (5) is small compared to $E$ ) for the limiting cases of weak and strong $F$.

In the weak-field limit, $F<<1$, we obtain from Eqs. (5) and (6):

$$
\epsilon=-1-\frac{1}{16} F^{2}\left[1+\frac{3}{2} F^{2}+\frac{7}{24}\left(1+13 F^{2}\right) \omega^{2}\right]-\frac{\mathrm{i}}{4} F\left[1+\frac{4}{45} \frac{\omega^{2}}{F^{3}}\right] \mathrm{e}^{-\frac{4}{3 F}}
$$

As may be seen from Eq. (7), a PT-account of $\omega \hat{L}_{z}$ in weak fields is possible only for small $\omega\left(\omega^{2}<F^{3}<<1\right)$, so that the frequency correction to the tunnelling pre-exponential factor is small. The Stark-shift, $\operatorname{Re} \epsilon+1$, given by Eq. (7) coincides exactly with the first two terms of the $\omega$-expansion for the known dynamic polarizability and hyperpolarizability of a weakly-bound particle [19]. The inapplicability of Eq. (7) for $\omega>1$ is evident already from the known fact that for weak fields and above-threshold frequencies the level width has the form of a power series in $F$. In particular, in the lowest PT order in $F$ (for an arbitrary polarization),

$$
\Gamma_{0}^{(1)}=\left(8 F^{2} / 3 \omega^{4}\right)(\omega-1)^{3 / 2}
$$

Finally, we note that $\operatorname{Im} \epsilon$ in Eq. (7) coincides with the first two terms of the expansion (in $\omega^{2}$ ) of the known result for the level width $\Gamma^{a d}$ for a circularly-polarized field in the adiabatic (Keldysh) approach [12]:

$$
\Gamma^{a d}=\frac{F}{2} \exp \left[-\frac{4}{3 F}\left(1-\frac{\omega^{2}}{15 F^{2}}\right)\right] .
$$

In the limit $F>>1$, Eqs. (5) and (6) give the following result:

$$
\begin{aligned}
\epsilon & =F^{2 / 3}\left\{0.44133122 \mathrm{e}^{-\mathrm{i} \frac{\pi}{3}}-\left[0.86328690 \mathrm{e}^{-\mathrm{i} \frac{\pi}{6}}-0.02548960 \mathrm{e}^{\mathrm{i} \frac{\pi}{3}} \frac{\omega^{2}}{F^{3}}\right] F^{-1 / 3}\right. \\
& \left.-0.3763512 F^{-2 / 3}+\ldots\right\}
\end{aligned}
$$

showing that in ultrastrong fields a perturbative account of the term $\omega \hat{L}_{z}$ is possible for any frequencies satisfying the condition $\omega^{2}<F^{3}$. (It is 


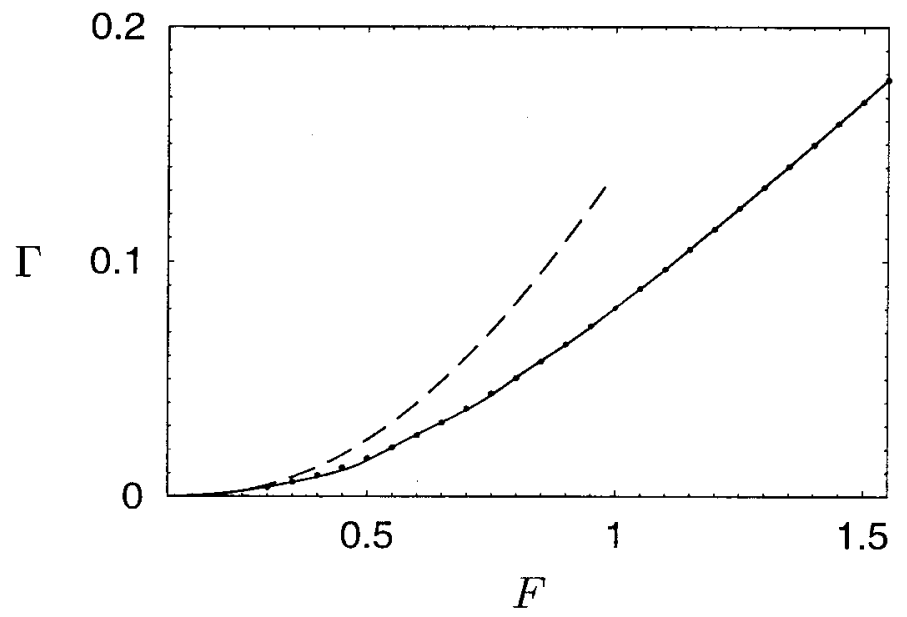

Figure 3. Dependence of $\Gamma$ on $F$ for a circularly polarized laser field having $\omega=0.74$. Solid curve: exact (numerical) result from Eq. (2); dashed curve: the weak-field approximation, Eq. (7); solid circles: the PT result in $\omega$, Eq. (5).

interesting that this condition is the reverse of that for the convergence of the standard PT in $F$ for the complex quasienergy [20]: $\Delta \equiv F^{2} / \omega^{3}<$ 1.) Although the asymptotic expansion of the energy $E$ in a static field, which was used in the derivation of $(10)$, is valid only for ultrastrong fields $(F>>10)[18]$, the result $(10)$ is noteworthy since it shows analytically the breakdown of QS in ultrastrong fields.

Concerning the applicability of the approximate result (5), we note that for $\omega<1$ it agrees well with the exact result (cf. Eq. (2)) both for moderate and strong fields. Fig. 3 shows that the exact curve for $\Gamma(F)$ at $\omega=0.74$ and that calculated using Eq. (5) are almost indistinguishable for $F \geq$ 0.5. Moreover, the weak-field (tunnelling) approximation, Eq. (7), is not applicable in this region of $F$, which corresponds to "overbarrier" ionization for the case of an atomic potential. It is interesting that, beginning from $F \approx 1$, the width $\Gamma$ exhibits actually a linear dependence on $F$ similar to the so-called "intermediate" asymptotic for $\Gamma(F)$ for overbarrier decay in a Coulomb potential [21]. In other words, for $\omega<1$ the action of a strong circularly-polarized field is equivalent to the action of a strong static field $\mathbf{F}$, and it is reasonably described by the analytic result (5).

For $\omega>1 \mathrm{PT}$ in $\omega \hat{L}_{z}$ is applicable only for ultrastrong fields. Thus results for the domain of stabilization and for $F \geq F_{c r}$ may be obtained only numerically. In Fig. 4 we compare the strong-field asymptote, from Eq. (10), with the exact (numerical) result for $\omega=1.55$. Although the range of $F$ considered is not enough to achieve close coincidence of the solid and dashed curves, the agreement improves as $F$ increases. Fig. 4 demonstrates also 


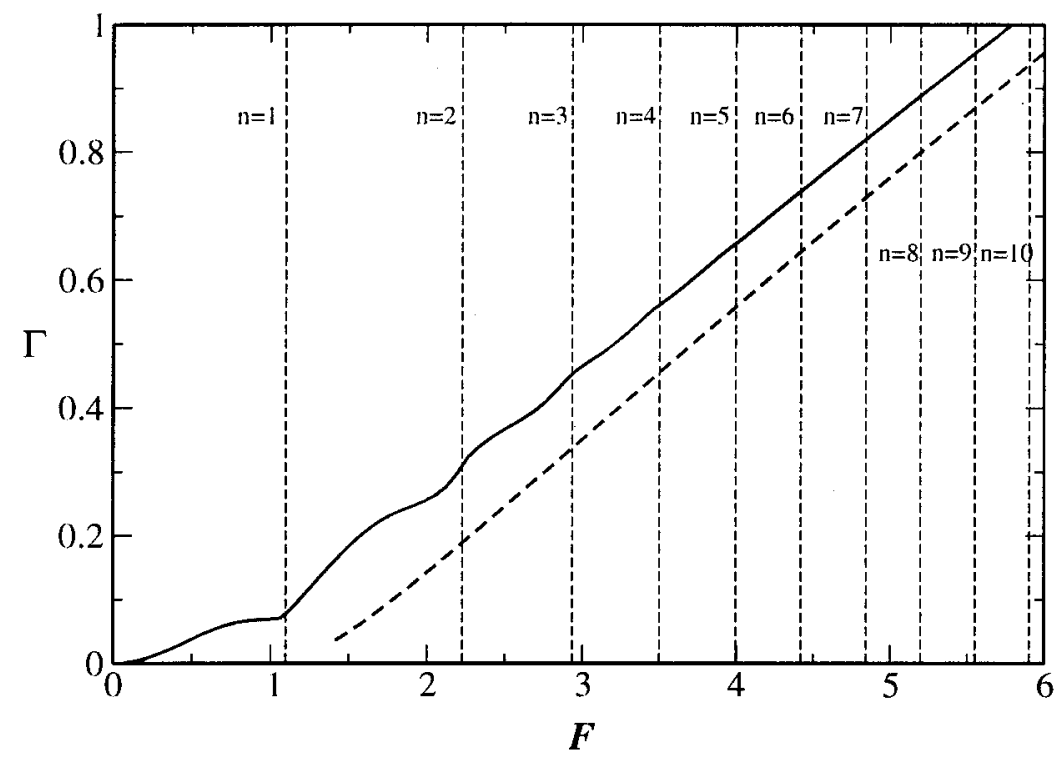

Figure 4. Comparison of exact (solid line) and approximate (dotted line) results for $\Gamma$ [from Eqs. (2) and (10)]for strong, circularly polarized fields with $\omega=1.55$. Vertical dashed lines denote threshold values of $F$, at which $\operatorname{Re} \epsilon-\mathcal{E}_{F}+\mathrm{n} \omega=0$.

threshold peculiarities for higher ATI-channels with $n>1$, although these peculiarities are smoothed out compared with $n=1$ (see also $[13,14]$ ). Our detailed calculations show that, for linear polarization, high-order threshold peculiarities are more pronounced than for circular polarization (see also [15]). Moreover, the stabilization-like regime (up to the closure of the direct photoionization channel) presented in Fig. 1 for $l=1$ exists also for $\omega<1$, although both the interval of QS (in $F$ ) and the variation of $\Gamma$ in this interval decrease rapidly with decreasing $\omega$.

\section{Discussion and conclusions}

Numerical and analytical analyses allow one to understand the major peculiarities of the quasistationary decay of a weakly-bound level for various relations among the key parameters, $\left|E_{0}\right|, \omega$, and $F$. Since (in contrast to the case of a static field) $F=0$ is the point of analyticity for the function $\epsilon=\epsilon(F)$, for $\Delta<<1$ the multiphoton (or perturbative) regime for decay is realized for any $F$ and $\omega$ including the case of $\omega<<1$, i.e., $\Gamma \sim F^{2 N}$ with $N=\left[\left|E_{0}\right| / \omega\right]$. For increasing $F$ the situation depends qualitatively on the frequency: for small $\omega$ the relation $\Delta \sim 1$ is achieved at relatively weak values of $F$, and the PT-regime merges smoothly into the tunnelling regime of Keldysh (see Eq. (9) for the case of circular polarization). This mecha- 
nism is realized for fields that are weak compared to interatomic ones (see Eq. (7) and the dashed curve in Fig.1). It has been analyzed in detail for arbitrary $\gamma[22]$. For higher $F$ (and small $\omega$ ) the tunnelling regime merges into the "overbarrier" regime (the solid curve in Fig. 3), which exists up to the passing of the quasistationary level into continuum.

For $\omega>1$ the decay mechanism is multiphotonic even for fields at which the first nonvanishing PT-result (see Eq. (8) for the case of a short-range potential) is already inapplicable. Hence it is necessary to take into account high-order corrections, which correspond to the re-emission of absorbed photons and to direct $n$-photon, above-threshold absorption. However, in this domain the linear dependence of $\Gamma$ on the intensity [see Eq. (8)] is changed: the correction of order $F^{2}$ to $\Gamma_{0}^{(1)}$ is negative [19]. Thus, the QS regime (in which $\Gamma$ no longer increases or even decreases with $F$ ) arises instead of the tumnelling regime. Its beginning corresponds to fields $F<$ $\omega$, at which higher-order PT-corrections become important. Obviously in the domain of an 'established' stabilization, PT in $F$ is inapplicable. In contrast to the low-frequency case, where Coulomb effects change only the pre-exponent factor in the tunnelling regime or the slope of the linear in $F$ dependence of $\Gamma$ for overbarrier decay, the QS regime is much more sensitive to the details of the potential. For a short-range potential, the Stark-shift is negative in the QS interval and the breakdown of QS is connected with the closure of the direct photoionization channel (see Figs. 1 and 4) and later with the passing of the quasibound state into the continuum for superstrong $F$. For a Coulomb potential, the Stark-shift is positive for $\omega>1$ and moderate $F$. Thus the closure of the photoionization channel is impossible here, and only the passing of the bound level into the continuum may lead to the breakdown of QS. But detailed analysis of this interesting question on the breakdown of QS for the Coulomb potential has not yet been performed.

In conchusion, our analysis demonstrates the existence of a $Q S$ regime for a weakly-bound particle at above-threshold frequencies, but only for a limited interval of laser intensities, whose upper limit, $F_{c r}$, is estimated analytically. The $\delta$-model potential describes photoprocesses in negative ions reasonably, especially for $\mathrm{H}^{-}$. Fig. 1 shows that (using the $\mathrm{Nd}$ :YAG

laser) the observation of $\mathrm{QS}$ for $\mathrm{H}^{-}$is possible at $F \sim 1\left(I \approx 3 \times 10^{12}\right.$ $\mathrm{W} / \mathrm{cm}^{2}$ ). Since the lifetime of $\mathrm{H}^{-}$in such a field is small, $\tau \sim 1 / \Gamma \sim 10 \mathrm{fs}$, stabilization may be observable using femtosecond pulses.

\section{Acknowledgments}

The authors are grateful to M.V.Fedorov for useful discussions. This research is supported in part by Grants INTAS-RFBR (No. 97-673), RFBR (No. 00-02-17843), and NSF (No. PHY-0070980). 
* On leave from Institute for Space Sciences, Bucharest-Măgurele 76900, Romania.

\section{References}

1. M.Pont and M.Gavrila, Phys. Rev. Lett. 65, 2362 (1990); M.Gavrila, in AIP Conference Proceedings 525, Multiphoton Processes: ICOMP VIII, 8th Interantional Conference, ed. by L.F.DiMauro, R.R.Freeman, and K.C.Kulander (Melveille, New York, 2000) p.103.

2. M.V.Fedorov and A.M.Movsesian, J. Phys. B 21, L155 (1988); M.V.Fedorov, Laser Phys. 9, 209 (1999).

3. Q.Su, Laser Phys. 2, 241 (1993); Q.Su, B.P.Irving, C.W.Johnson, and J.H.Eberly, J. Phys. B 29, 5755 (1996).

4. Q.Su, B.P.Irving, and J.H.Eberly, Laser Phys. 7, 1 (1997); A.Patel, N.J.Kylstra, and P.L.Knight, J. Phys. B 32, 5759 (1999); A.M.Popov, O.V.Tikhonova, and E.A.Volkova, Laser Phys. 10, 779 (2000).

5. M.Dorr and R.M.Potvliege, J. Phys. B 33, L233 (2000).

6. V.P.Krainov and M.A.Preobragenskii, Zh. Exp. Teor. Fiz. 103, 1142 (1993) [Sov. Phys. JETP 76, 559 (1993)].

7. J.Z.Kamiński, Phys. Rev. A 52, 4976 (1995).

8. S.Geltman, J. Phys. B 32, 853 (1999); T.Mercouris and C.Nicolaides, ibid. 32, 2371 (1999); C.Figueira, A.Fring, and R.Schrader, Laser Phys. 9, 379 (1999).

9. N.L.Manakov, M.V.Frolov, B.Borca, and A.F.Starace, Pis'ma ZETF, 72, 426 (2000) [JETP Lett. 72, $294(2000)]$.

10. N.L.Manakov, V.D.Ovsiannikov, and L.P.Rapoport, Phys. Rep. 141, 319 (1986).

11. N.L.Manakov and A.G.Fainshtein, Dokl. Akad. Nauk SSSR, 244, 567 (1979) [Sov. Phys. Dokl. 24, 41 (1979)]; Zh. Exp. Teor. Fiz. 79, 751 (1980) [Sov. Phys. JETP 52, $382(1980)]$.

12. N.L.Manakov and L.P.Rapoport, Zh. Exp. Teor. Fiz. 69, 842 (1975) [Sov. Phys. JETP 42, 430 (1975)]; I.J.Berson, J. Phys. B 8, 3078 (1975).

13. W.Becker, J.K.McIver, and M.Confer, Phys. Rev. A, 406904 (1989); W.Becker, S.Long, and J.K.McIver, ibid. 424416 (1990);

14. F.H.M.Faisal, P.Filipowicz, and K.Rzazewski, Phys. Rev. A 41, 6176 (1990); P.S. Krstić, D. B. Milošević, and R. K. Janev, Phys. Rev. A 44, 3089 (1991).

15. P. Filipowicz, F.H.M.Faisal, and K.Rzazewski, Phys. Rev. A 44, 2210 (1991).

16. M.Dorr, R.Potvliege and R.Shakeshaft, Phys. Rev. Lett. 64, 2003 (1990).

17. Ya.B.Zel'dovich, Zh. Exp. Teor. Fiz. 39, 776 (1961) [Sov. Phys. JETP 12, 542 (1961)].

18. N.L.Manakov, M.V.Frolov, A.F.Starace, and I.I.Fabrikant, J. Phys. B 33, R,141 (2000).

19. N.L.Manakov, L.P.Rapoport, M.A.Preobragenskii, and A.G.Fainshtein, Zh. Exp. Teor. Fiz.75, 1243 (1978) [Sov. Phys. JETP 48, 626 (1978)].

20. N.L.Manakov and A.G.Fainshtein, Teor. Mat. Fiz. 48, 375 (1981) [Theor. Math. Phys. 48, 815 (1982)]; R.M.Potvliege and R.Shakeshaft, Phys. Rev. A 41, 1609 (1990).

21. V.S.Popov, Zh. Exp. Teor. Fiz.118, 56 (2000) [Sov. Phys. JETP 01, 48 (2000)].

22. L.V.Keldysh, Zh. Exp. Teor. Fiz. 47, 1945 (1964) [Sov. Phys. JETP 20, 1307 (1964)]; A.I.Nikishov and V.I.Ritus, ibid. 50, 255 (1966) [23, 168 (1966)]; A.M.Perelomov, V.S.Popov, and M.V.Terent'ev, ibid. 50, 1393 (1966) [23, 1924 (1966)]; 51, 309 (1966) $[\mathbf{2 4}, 207(1967)]$. 